\title{
La calidad de la democracia. Examen de sus fundamentos conceptuales a comienzos del siglo XXI ${ }^{1}$
}

\section{The quality of democracy. Examination of its concep ual basis at the beginning of the 21 st century}

Recibido: 10 de mayo de 2017- Revisado: 12 de septiembre de 2017 - Aceptado: 16 de octubre de 2017.

\section{Milton Andrés Rojas-Betancur ${ }^{2}$ Mauricio Bocanument-Arbeláez ${ }^{3}$ Dany Esteban Gallego-Quiceno ${ }^{4}$ María Angélica Pineda-Carreño ${ }^{5}$}

\section{Resumen}

Actualmente uno de los campos de mayor relevancia en el es ıdio de la democracia es el de su calidad. Por lo tanto, se formularon unos supuestos c ceptuales pr ra definirla que sirven como punto de partida y diálogo entro diversos a. res. Adi ionalmente, se realizó una revisión de literatura acerca del desa is v evolucivin uel concepto. El rastreo se realizó con el propósito de indagar acerc: de l. lidad de la democracia"

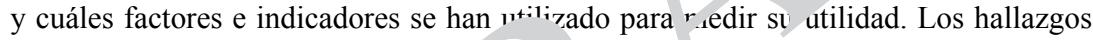
evidenciaron que los estudios sobre ‘a deı cracia pá ten de una definición operativa fundamentada en el concepto de poı quía que le ha rmitido delimitar su objeto de estudio y centrarse en aspectro instituc rales _. rostulados conceptuales a partir de por lo menos tres diferent ${ }^{+} \mathrm{s}$ רfoques de álisis.

Palabras clave

Calidad de la d snocracia, poliarq instituciones políticas, democratización.

\section{Abstract}

Curr .... me of . most ielevant fields in the study of democracy is its quality. $\mathrm{T}^{\prime}$ refore :onceptua sumptions were formulated to define it as a starting point and dia ue ween various authors. Additionally, a literature review was made about the develc nent alı evolution of this concept. The tracking was conducted to investigate about "4 lity of democracy" and which factors and indicators have been used to measure $i^{1}$ s usefulness. The findings showed that the studies on democracy start from an operative definition based on the concept of polyarchy, which has allowed it to delimit its object of study and focus on institutional aspects under conceptual postulates from at least three different analysis approaches.

\section{Keywords}

Quality of democracy, polyarchy, politics institutions, democratization.

\section{${ }^{1}$ Artículo de revisión.}

2 Abogado, Magister en Ciencia Política y $\mathrm{PhD}$. (c) en Ciencias Humanas y Sociales de la Universidad Nacional de Colombia. Profesor de tiempo completo del Departamento de Ciencias Sociales y Humanas de la Universidad de Medellín, Medellín, Colombia. Correos electrónicos: marojas@udem.edu.co; miltonrojasb@ gmail.com

${ }^{3}$ Abogado, Especialista en Derecho de Familia, Magíster en Gobierno y Doctor en Derecho de la Universidad de Medellín. Vicerrector Académico de la Corporación Universitaria de Sabaneta, Antioquia, Colombia. Integrante de CONACES del Ministerio de Educación Nacional de Colombia. Correos electrónicos: mbocanument@gmail.com; vicerrector.academico@unisabaneta.edu.

4 Licenciado, Máster en Investigación, Magíster en Educación y Máster en Administración de Negocios Internacionales, Doctorando en Ciencias de la Educación. Vicerrector Académico de la Corporación Universitaria Americana, Medellín, Colombia. Correo electrónico: danygallego@ yahoo.com

${ }^{5}$ Economista, Abogada, Estudiante de maestría en tributación, Investigadora de la Corporación Universitaria de Sabaneta, Antioquia, Colombia Correo electrónico: mariangelicapinedac@ hotmail.com

Para citar este artículo use: RojasBetancur, M., Bocanument-Arbeláez, M., Gallego-Quiceno, D., y PinedaCarreño, M. (2018). La calidad de la democracia. Examen de sus fundamentos conceptuales a comienzos del siglo XXI. Revista Civilizar, 18(34), 105-118. 